\title{
6th Grazer Risk Day: The Future of Yesterday in Healthcare
}

\author{
Graz, Austria, 5 April 2018
}

Published: 5 April 2018

A1

Critical incident reporting system in tertiary university hospital Gerald Sendlhofer ${ }^{1,2}$, Veronika Gombotz', Peter Tiefenbacher', Karina Leitgeb', Gernot Brunner ${ }^{2}$

'Executive Department for Quality and Risk Management, University Hospital Graz, Graz, Austria; ${ }^{2}$ Research Unit for Safety in Health, c/o Division of Plastic, Aesthetic and Reconstructive Surgery, Department of Surgery, Medical University of Graz, Graz, Austria

Correspondence: Gerald Sendlhofer (gerald.sendlhofer@klinikum-graz.at) Safety in Health 2018, 4(Suppl 1):A1

Key words: Critical incident, CIRS, patient safety, employee safety Background

Within our organization the critical incident reporting system (CIRS) was introduced in 2013. Since then, only limited use can be recognized in terms of how many CIRS-cases were reported to the onlineavailable system. Reasons for underreporting are divers, however, dominantly it's the fear of healthcare workers (HCW) whether the anonymity of the reporting employee is preserved. Consequently, we analyzed CIRS-publication data from 2013 to 2017 in order to interpret the development of CIRS in a tertiary university hospital.

Material and methods

Based on reported CIRS-cases since the introduction of the onlineavailable system, data were analyzed according to the total number of reported CIRS-cases, who reported a CIRS-case, which specialty used CIRS and finally what kind of risk was reported most often. We also distinguished if the reported CIRS-case was in term of our definition a correct CIRS-case or a so-called near miss (relevant for employee safety) or if it was a patient harm.

Results

In total 683 cases were reported into the CIRS, of which only 283 had been a correct CIRS-case. 400 cases had been no CIRS or near miss case. Predominantly, CIRS-cases concerning surgical processes were reported (16\%), followed by CIRS-cases concerning medicinal products (16\%) and CIRS-cases concerning medical devices (10\%). It was more likely, that nurses reported into CIRS (44\%) than by physicians (24\%). $45 \%$ of all CIRS-cases were reported by surgical disciplines, $21 \%$ by interdisciplinary organization, $16 \%$ by non-surgical disciplines and $13 \%$ came from anaesthesiology.

\section{Conclusions}

A CIR-system is an essential component of clinical risk management and is a simple tool that can be used to identify potential sources of critical incidents (1). CIRS can be a valuable instrument if CIRS is used as foreseen. As CIRS is used just in rare situations according to the presented data, CIRS should be introduced more comprehensively to convince HCW that reporting into CIRS is safe and that any CIRS-case can help to avoid future harm to patients.

\section{Competing interests}

The authors have no competing interests. There was no funding.

\section{References}

1) Sendlhofer G, Eder H, Leitgeb K, Gorges R, Jakse H, Raiger M, et al. Survey to identify depth of penetration of critical incident reporting systems in Austrian healthcare facilities. Inquiry, 2018; doi: 10.1177/0046958017744919
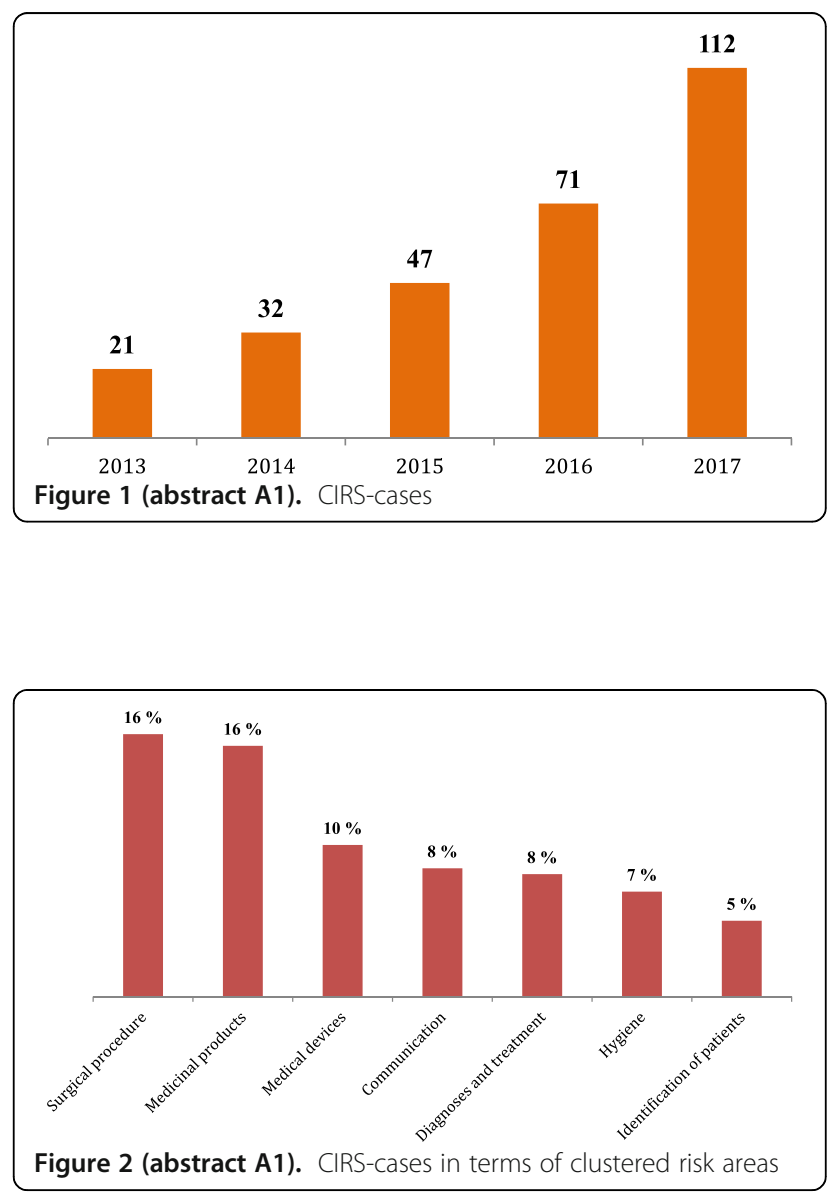
A2

A new instrument to identify quality and safety aspects in healthcare organizations: self-assessment versus an assessment by independent experts

Christian Richter', Gerald Sendlhofer ${ }^{1,2}$, Susanne Gasteiner', Gernot Brunner ${ }^{2}$ 'Executive Department for Quality and Risk Management, University Hospital Graz, Graz, Austria; ${ }^{2}$ Research Unit for Safety in Health, c/o Division of Plastic, Aesthetic and Reconstructive Surgery, Department of Surgery, Medical University of Graz, Graz, Austria

Correspondence: Christian Richter (Christian.richter@klinikum-graz.at) Safety in Health 2018, 4(Suppl 1):A2

Key words: Quality, patient safety, self-assessment, criteria catalogue Background

Within our organization mainly two quality standards are used. In the past, ISO 9001 certification as well as the EFQM-model had been introduced within 8 out of 20 clinical departments. Furthermore, each department is externally audited concerning clinical risk management in a four year interval. However, there is no hospital-wide system available that assures a minimum quality and patient safety standard. Therefore, it was the aim to develop a minimum criteria set that can be used to identify and prove the quality and safety standard in each department. Furthermore, the criteria set also contains further items to identify departments with an advanced quality and patient safety standard.

Material and methods

Based on ISO 9001, EFQM and in-house standards, a catalogue with a set of six categories and in total 60 criteria were developed. In each category so-called "must-have" items were determined which represents the minimum level. Add-on criteria are divided in "bronze", "silver" and "gold" status. In theory, departments with an ISO-certification should reach the "silver" level, whereas EFQM-departments should be able to reach the "gold" level. The first step is the self-assessment which has to be carried out by an interdisciplinary team of physicians, nurses, medical technical assistants and quality and risk managers. Healthcare workers with and without managerial function perform the self-assessment and rate each of the 60 criteria. Thereafter, the result has to be forwarded to the Department of Quality and Risk Management. They perform an independent assessment in terms of evaluating the answers given in the self-assessment by performing an internal audit.

Results

Six categories were assembled. In each of the categories four criteria were the same (key indicator, communication/information/documentation, risks and chances, avoiding waste). Underlined criteria reflect the "must haves".

In category 1 the following criteria are available: 1 ) legal basis for the management of the organization, 2) organization chart, functional description, delegation, 3) interested groups, 4) vision, policy, 5) strategy and goals, 6) reporting, 7) leadership principles.

In category 2 the following criteria are available: 1 ) health promotion, 2) prevention, 3) crisis management, 4) idea management, 5) introduction and discharge of employees, 6) advanced education, 7) staff appraisal In category 3 the following criteria are available: 1) risk-management process, 2) patient safety relevant guidelines, 3) patient identification, 4) hand hygiene, 5) pain management, 6) medication safety, 7) multidisciplinary tumor boards, 8) discharge management, 9) CIRS and complaints, 10) patient empowerment

In category 4 the following criteria are available: 1) processes, 2) process map, 3) document management

In category 5 the following criteria are available: 1 ) interested groups and partners, 2) customer, 3) referring physician, 4) supplier and partners In category 6 the following criteria are available: 1) knowledge management, 2) medicinal products and medical devices, 3) ethical policy, 4) human resource planning, 5) data protection

\section{Conclusions}

A set of criteria was developed to assess quality and patient safety standards in a tertiary university hospital. Reasons for the development had been that ISO 9001 and EFQM standards were not designed in a patient centered way. These standards regulate how organizations should function in principle, however, in a healthcare setting these standards are often not practical and or in other words do not reach each healthcare worker and are not affecting patient safety.

Competing interests

The authors have no competing interests. There was no funding.

Table 1 (abstract A2). Categories

\begin{tabular}{ll}
\hline Category & Number of items \\
\hline Interdisciplinary Leadership & 11 \\
Staff & 11 \\
Patient Safety & 14 \\
Processes & 7 \\
Interest Group and Partners & 8 \\
Resources & 9 \\
\hline
\end{tabular}

A4

Evaluation of documentation and treatment quality of a new implemented insulin chart at a large university hospital

Julia Kopanz', Katharina M. Lichtenegger', Gerald Sendlhofer ${ }^{2,3}$, Barbara Semlitsch', Regina Riedl ${ }^{4}$, Thomas R. Pieber', Christa Tax ${ }^{5}$, Gernot Brunner ${ }^{3,5}$, Johannes Plank ${ }^{6}$

'Division of Endocrinology and Diabetology, Department of Internal Medicine, Medical University of Graz, Graz, Styria, Austria; ${ }^{2}$ Department of Quality and Risk Management, University Hospital Graz, Graz, Styria, Austria; ${ }^{3}$ Research Unit for Safety in Health, Division for Plastic, Aesthetic and Reconstructive Surgery, Department for Surgery, Medical University of Graz, Graz, Styria, Austria; ${ }^{4}$ Institute for Medical Informatics, Statistics and Documentation, Medical University of Graz, Graz, Styria, Austria; ${ }^{5}$ Board of Directors, University Hospital Graz, Graz, Styria, Austria;

${ }^{6}$ Division of Gastroenterology and Hepatology, Department of Internal

Medicine, University Hospital Graz, Graz, Styria, Austria

Correspondence: Julia Kopanz (julia.kopanz@medunigraz.at)

Safety in Health 2018, 4(Suppl 1):A4

Key words: insulin chart, quality, inpatient, insulin error Background

Insulin charts are a common tool for documentation, communication, interpretation, insulin prescription and insulin administration in the diabetic inpatient setting [1]. As listed as high-alert medication [2] insulin can cause harm when not used correctly $[3,4]$. Especially errors in insulin prescription and insulin administration are reported in current literature [3-7]. Due to the high heterogeneity of 20 different insulin charts at the University Hospital Graz, Austria, a new standardized paper-based insulin chart (Figure 1) was generated [8]. The new insulin chart was implemented as a pilot project in the Department of Internal Medicine. Our aim was to evaluate filled-in insulin charts regarding documentation and treatment quality and to conduct a before-aftercomparison of the filled-in old and new insulin charts.

Materials and Methods

The evaluation took place at the general inpatient wards of the Department of Internal Medicine. Filled-in insulin charts of all 
patients who were treated with insulin and who were admitted to each ward for at least 24 hours were evaluated using a paper-based evaluation form based on the methodology of the National Diabetes Inpatient Audit (NDIA) [9-11]. Depending on availability up to 15 old and 15 new insulin charts were collected per ward. All data were pseudonymized and analyzed using IBM SPSS Statistics 23 .

Results

Overall 108 old and 100 new insulin charts were evaluated. The documentation and treatment quality of the filled-in new insulin charts improved compared to the filled-in old insulin charts. Fewer errors occurred for all listed parameters (Table 1). Most importantly, new insulin charts had nearly half of the number of insulin errors relative to the old insulin charts. Especially signing of insulin prescriptions was much more frequent on new insulin charts (58\% vs. $0 \%)$ but signatures were still missing on $42 \%$ of new insulin charts.

\section{Conclusions}

Through implementation of the new insulin chart great improvements in documentation and treatment quality were achieved. Most likely, these improvements were caused by structural diversity of old and new insulin chart. Nevertheless, as some errors still occur, further potential for improvement should be derived from the results to optimize quality and safety of inpatient diabetes care.

\section{Acknowledgements}

The authors thank all the participating departments for their collaboration.

\section{Competing Interests}

There are no competing interests.

\section{Reference List}

1. Christofidis MJ, Horswill MS, Hill A, McKimmie BM, Visser T, Watson MO. Task Analysis and Heuristic Analysis of Insulin Charts: Final report prepared for the Australian Commission on Safety and Quality in Health Care: 2 February 2012. [http://www.safetyandquality.gov.au/wp-content/ uploads/2012/06/56679-Insulin-charts-heuristic-analysis-2-Feb-2011-FinalReport.pdf] accessed January 23, 2018.

2. Institute for Safe Medication Practices (ISMP). List of high-alert medications in acute care settings. 2014. [https://www.ismp.org/tools/highalertmedications.pdf] accessed January 23, 2018.

3. Fowler D, Rayman G. Safe and effective use of insulin in hospitalised patients. London: NHS; 2010. 16 p.

4. Cox AR, Ferner RE. Prescribing errors in diabetes. Br J Diabetes Vasc Dis. 2009; 9:84-88.

5. Rushmer R, Voigt D. Measure it, improve it: the safer patients initiative and quality improvement in subcutaneous insulin therapy for hospital inpatients. Diabet Med. 2008; 25:960-967.

6. National Diabetes Inpatient Audit. National Diabetes Inpatient Audit England and Wales, 2016. Full report. 2017. [https://digital.nhs.uk/ catalogue/PUB23539] accessed January 23, 2018.

7. Lamont T, Cousins D, Hillson R, Bischler A, Terblanche M. Safer administration of insulin: summary of a safety report from the National Patient Safety Agency. BMJ. 2010; 341:882-887.

8. Plank J. Identifikation von Qualität und Risiken der Diabeteskurven zur Optimierung der Diabetesversorgung im LKH-Univ. Klinikum Graz - Diab_Curve [master's thesis]. Graz (AUT), Medical University of Graz 2015: 104 p.

9. National Diabetes Inpatient Audit. National Diabetes Inpatient Audit 2013. National summary. 2014. [https://digital.nhs.uk/catalogue/ PUB13662] accessed January 23, 2018.

10. National Diabetes Inpatient Audit. Hospital Characteristics Questionnaire. 2013. [http://www.hscic.gov.uk/diabetesinpatientaudit] accessed January 23, 2018.

11. National Diabetes Inpatient Audit. Bedside Audit Questionnaire. 2013. [http://www.hscic.gov.uk/diabetesinpatientaudit] accessed January 23, 2018

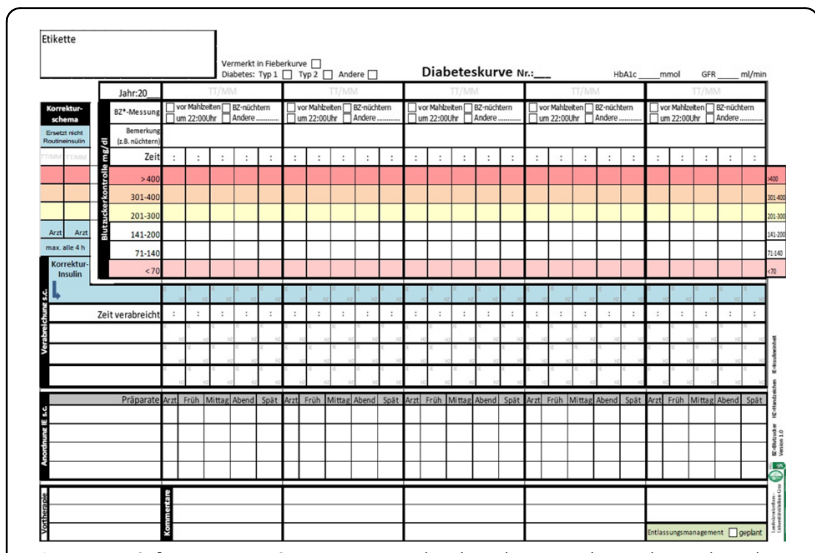

Figure 1 (abstract A4). New standardized paper-based insulin chart of the University Hospital Graz

Table 1 (abstract A4). Main results of documentation and treatment quality evaluation of filled-in old and new insulin charts at the Department of Internal Medicine, University Hospital Graz

\begin{tabular}{|c|c|c|}
\hline Insulin charts with ... & $\begin{array}{l}\text { old insulin charts } \\
(n=108)\end{array}$ & $\begin{array}{l}\text { new insulin charts } \\
(n=100)\end{array}$ \\
\hline correct patient identification & $84(78)$ & $90(90)$ \\
\hline written up insulin & $81(75)$ & $100(100)$ \\
\hline signed insulin prescription & 0 & $58(58)$ \\
\hline signed insulin administration & $53(49)$ & $97(97)$ \\
\hline $\begin{array}{l}\text { documented time of insulin } \\
\text { administration }\end{array}$ & $62(57)$ & $100(100)$ \\
\hline $\begin{array}{l}\text { documented hypoglycemia treatment } \\
(54-72 \mathrm{mg} / \mathrm{dl})\end{array}$ & $\begin{array}{l}3(16) \text { out of } 19 \\
(100)\end{array}$ & $\begin{array}{l}5(31) \text { out of } 16 \\
(100)\end{array}$ \\
\hline
\end{tabular}

Data are presented as $\mathrm{n}(\%)$

A5 Attitude towards the surgical safety checklist: a question of gender, age or profession?

Christian Smolle $e^{1,2}$, Gerald Sendlhofer ${ }^{1,3}$, Gernot Brunner ${ }^{4}$, Lars-Peter Kamolz ${ }^{1,2}$ ${ }^{1}$ Research Unit Safety in Health, Division of Plastic, Aesthetic and Reconstructive Surgery, Department of Surgery, Medical University of Graz, Graz, Austria; 'Division of Plastic, Aesthetic and Reconstructive Surgery, Department of Surgery, Medical University of Graz, Graz, Austria; ${ }^{3}$ Executive Department for Quality and Risk Managment, University Hospital Graz, Graz, Austria; ${ }^{4}$ Chief Medical Director, University Hospital Graz, Graz, Austria

Correspondence: Christian Smolle (christian.smolle@medunigraz.at) Safety in Health 2018, 4(Suppl 1):A5

Key words: Surgical Safety Checklist, patient safety, safety, compliance, attitude Introduction

The surgical safety checklist (SSC) has been seen to reduce postoperative complications, but its effective use seems to be highly dependent on the attending staff's compliance and attitude towards it. The aim of this study was to evaluate the attitude of 
the operative staff towards the SSC and whether differences existed between nurses and physicians.

Methods

Members of the operative nursing staff and physicians were asked to participate in an online-survey. Basic parameters, including age, gender, years of working experience and average estimated time spent with patients within one working week were assessed. The questionnaire consisted of 26 statements regarding pros and cons and estimated importance of the SSC. The statements could be rated on a Likert scale ranging from 1 (totally agree) to 7 (do not agree at all). Answers of nurses and physicians were compared to one another. To detect any possible bias caused by differences of basic parameters between the two groups, univariate analysis $\left(\mathrm{Chi}^{2}, \mathrm{~T}\right.$-test, Spearmancorrelation) was followed by linear regression models. A p-value $<0.05$ was considered as statistically significant.

Results

In total, 327 (136 physicians, 191 nurses) questionnaires were returned. Nurses were on average younger than physicians (36.7 vs. 44.9 years, $p<0.001)$ and there were more women among the nursing staff $(79.6 \%$ vs. $38.2 \%, p<0.001)$. Nurses claimed to spend more time with patients $(p<0.001)$ but had less working experience $(p=0.014)$. In 4 of the 26 survey items there were significant differences between nurses and physicians (Table 1$)$. Physicians claimed more often they could use the SSC correctly $(p=0.049)$ and that they had influence on its correct use, whereas nurses tended to state that the SSC was not taken seriously by surgeons $(p=0.001)$ and that their colleagues took its use seriously.

Linear regression models including all basic parameters showed that the results were not solely dependent on profession but (co-)influenced by age, gender, and years of working experience (Table 2).

\section{Discussion}

The survey revealed a positive attitude towards the SSC by both nurses and physicians. However, differences between professions exist: Nurses estimated the importance surgeons attribute to the SSC lower than physicians and physicians were less likely to state that their colleagues took the SSC seriously - a finding which could be interpreted as confession. On the other hand experienced female physicians seem to be most self-confident regarding their influence on the correct use of the SSC.

Table 1 (abstract A5). Answers of nurses and physicians; medians and interquartile range of the 7-item Likert-scale, n.s. = not significant

\begin{tabular}{|c|c|c|c|}
\hline Item & $\begin{array}{l}\text { Nurses, } \\
\mathrm{n}=191\end{array}$ & $\begin{array}{l}\text { Physicians, } \\
\mathrm{n}=136\end{array}$ & $\begin{array}{l}\text { Spearman-correlation, } \\
\text { p-value }\end{array}$ \\
\hline The SSC is evidence based. & $2[1-4]$ & $2[1-3]$ & n.s. \\
\hline The SSC is just a tick-off list. & $7[4-7]$ & $6[4-7]$ & n.s. \\
\hline I can use the SSC correctly. & $1[1-2]$ & $1[1-2]$ & 0.049 \\
\hline $\begin{array}{l}\text { The SSC makes it easier to utter } \\
\text { safety concerns in the OR. }\end{array}$ & $2[1-3]$ & $2[1-3]$ & n.s. \\
\hline $\begin{array}{l}\text { I advocate the use of the SSC in my } \\
\text { team. }\end{array}$ & $1[1-3]$ & $1[1-2]$ & n.s. \\
\hline $\begin{array}{l}\text { The SSC is unnecessarily time- } \\
\text { consuming. }\end{array}$ & $6[6-7]$ & $7[5-7]$ & n.s. \\
\hline $\begin{array}{l}\text { I will advocate the use of the SSC at } \\
\text { my work place. }\end{array}$ & $1[1-3]$ & $1[1-2]$ & n.s. \\
\hline $\begin{array}{l}\text { I will help my colleagues with the } \\
\text { use of the SSC. }\end{array}$ & $1[1-2]$ & $1[1-2]$ & n.s. \\
\hline The SSC reduces adverse events. & $1[1-2]$ & $1[1-2]$ & n.s. \\
\hline $\begin{array}{l}\text { The next time I face the decision, I } \\
\text { am going to use the SSC. }\end{array}$ & $1[1-2]$ & $1[1-2]$ & n.s. \\
\hline $\begin{array}{l}\text { I intend to take care of the correct } \\
\text { use of the SSC. }\end{array}$ & $1[1-1]$ & $1[1-1]$ & n.s. \\
\hline $\begin{array}{l}\text { I would like the SSC to be used } \\
\text { when I undergo surgery. }\end{array}$ & $1[1-1]$ & $1[1-2]$ & n.s. \\
\hline
\end{tabular}

Table 1 (abstract A5). Answers of nurses and physicians; medians and interquartile range of the 7-item Likert-scale, n.s. = not significant (Continued)

\begin{tabular}{|c|c|c|c|}
\hline Item & $\begin{array}{l}\text { Nurses, } \\
\mathrm{n}=191\end{array}$ & $\begin{array}{l}\text { Physicians, } \\
\mathrm{n}=136\end{array}$ & $\begin{array}{l}\text { Spearman-correlation, } \\
\text { p-value }\end{array}$ \\
\hline $\begin{array}{l}\text { The SSC complicates information } \\
\text { exchange in the OR. }\end{array}$ & $7[6-7]$ & $7[6-7]$ & n.s. \\
\hline $\begin{array}{l}\text { The SSC is a valuable tool for patient } \\
\text { safety. }\end{array}$ & $1[1-2]$ & $1[1-2]$ & n.s. \\
\hline $\begin{array}{l}\text { The SSC is not taken seriously } \\
\text { among surgeons. }\end{array}$ & $3[2-4]$ & $4[2-6]$ & 0.001 \\
\hline $\begin{array}{l}\text { I would like to see the SSC to be } \\
\text { used in all patients. }\end{array}$ & $1[1-1]$ & $1[1-1]$ & n.s. \\
\hline $\begin{array}{l}\text { I contribute to the correct use of the } \\
\text { SSC. }\end{array}$ & $1[1-2]$ & $1[1-2]$ & n.s. \\
\hline $\begin{array}{l}\text { My colleagues take the use of the } \\
\text { SSC seriously. }\end{array}$ & $1[1-2]$ & $2[1-3]$ & 0.031 \\
\hline $\begin{array}{l}\text { The SSC increases awareness for } \\
\text { patient safety. }\end{array}$ & $1[1-2]$ & $1[1-2]$ & n.s. \\
\hline The SSC interferes with my tasks. & $7[6-7]$ & $7[6-7]$ & n.s. \\
\hline $\begin{array}{l}\text { Good surgical staff does not need } \\
\text { the SSC. }\end{array}$ & $2[1-4]$ & $7[6-7]$ & n.s. \\
\hline $\begin{array}{l}\text { I have influence on the correct use } \\
\text { of the SSC in my team. }\end{array}$ & $2[1-4]$ & $1[1-2]$ & 0.002 \\
\hline $\begin{array}{l}\text { My superiors advocate the use of the } \\
\text { SSC. }\end{array}$ & $1[1-2]$ & $1[1-2]$ & n.s. \\
\hline $\begin{array}{l}\text { I am being expected to use the SSC } \\
\text { correctly. }\end{array}$ & $1[1-1]$ & $1[1-1]$ & n.s. \\
\hline $\begin{array}{l}\text { I see it as my task to use the SSC } \\
\text { correctly }\end{array}$ & $1[1-1]$ & $1[1-1]$ & n.s. \\
\hline The SSC is not needed. & $7[7-7]$ & $7[6-7]$ & n.s. \\
\hline
\end{tabular}

Table 2 (abstract A5). Results of linear regression analysis

\begin{tabular}{lll}
\hline Item & Influential parameters & p-value \\
\hline I can use the SSC correctly. & Higher age & 0.039 \\
The SSC is not taken seriously & Nursing staff & 0.013 \\
among surgeons. & & \\
My colleagues take the use of & Higher age & 0.016 \\
the SSC seriously. & Nursing staff & 0.027 \\
I have influence on the correct use & Female gender & 0.040 \\
of the SSC in my team. & $\begin{array}{l}\text { More working experience } \\
\text { Physician }\end{array}$ & 0.024 \\
& P.017
\end{tabular}

A6

Towards a digital WHO surgical safety checklist: problems and perceptions of users in a teaching hospital

K. Kiefel' ${ }^{1}$, K. Donsa' ${ }^{1}$ P. Tiefenbacher ${ }^{2}$, T.R. Pieber ${ }^{1,3}$, G. Sendlhofer ${ }^{2,4}$

'HEALTH - JOANNEUM RESEARCH Forschungsgesellschaft mbH, Graz, Austria; ${ }^{2}$ Executive Department for Quality and Risk Management, University Hospital Graz, Graz, Austria; ${ }^{3}$ Medical University of Graz, Graz, Austria; ${ }^{4}$ Research Unit for Safety in Health, c/o Division of Plastic, Aesthetic and Reconstructive Surgery, Department of Surgery, Medical University of Graz, Graz, Austria

Correspondence: Klaus Donsa (klaus.donsa@joanneum.at)

Safety in Health 2018, 4(Suppl 1):A6

Key words: digital checklist, electronic checklist, surgical safety checklist, WHO, surgery, quality management, digitalization Background

Although, the Surgical Safety Checklist (SSC) is nowadays standard in operating rooms (OR), their acceptance is often low. Evaluations of 
the currently in the University Hospital of Graz (Graz, Austria) used SSC showed, that especially the Team Time Out (TTO) and the Sign Out were not duly completed. New digital approaches of SSC showed promising improvements.

Objective

The aim of this project is to identify the problems associated with the SSC in daily routine and collect perceptions towards digitalization of the SSC from OR personnel.

\section{Material and methods}

Within this project, we analysed the perioperative processes associated with the SSC in the surgical departments. Therefore, we visited two OR and three surgery departments from the University Hospital of Graz (Graz, Austria). To identify user requirements, we conducted 12 semi-structured interviews with OR personnel. After the interviews, the participants filled out a short standardized questionnaire.

Results

The interview participants strongly agreed that the use of the SSC could prevent complications in the OR (75\%) and reduce errors if used properly (67\% "strongly applies", 33\% "applies"). The main problems identified in our observation were: (1) redundancy in material documentation and time tracking, (2) unprecise documentation of process times, (3) lack of attention and acceptance during the TTO process, (4) absence when TTO started and (5) problems with heterogeneous information sources (e.g. presence of allergies). Our evaluation showed that the majority of interview participants were satisfied with the current use of the SSC, but pointed out that a modification is necessary (7 out of 12). Nearly all of the participants had a positive attitude towards a digital SSC (10 out of 12) and more than half of participants desired a digitalized form of the checklist. A digital system (tablet and computer) linked to the Electronic Health Record (EHR) was the top choice (75\%) of interview participants.

\section{Conclusions}

The results demonstrate that the paper-based SSC is accepted but a digitalization is appreciated. A tablet-based client-server system with integration in EHR was preferred by the majority of participants.

\section{A7}

Risks of medical discharge documentation

Magdalena Hoffmann', Gerald Sendlhofer ${ }^{1,2}$, Petra Schwarz ${ }^{3}$, Gernot Brunner $^{2}$

${ }^{1}$ Executive Department for Quality and Risk Management, University Hospital Graz, Graz, Austria; ${ }^{2}$ Research Unit for Safety in Health, c/o Division of Plastic, Aesthetic and Reconstructive Surgery, Department of Surgery, Medical University of Graz, GrazAustria; ${ }^{3}$ Carinthia University of Applied Science, Feldkirchen, Austria

Correspondence: Magdalena Hoffmann

(Magdalena.hoffmann@klinikum-graz.at)

Safety in Health 2018, 4(Suppl 1):A7

Key words: Discharge letter, risk, patient safety

\section{Background}

The medical discharge letter (so-called doctor's letter) is the most important means of communication between hospitals and other healthcare providers. As a "medical condensate", it is of central importance for referring general practitioners as well as for other healthcare providers responsible for continued care of the patients. Despite its high status, it often happens that the discharge letter does not meet the desired requirements in everyday clinical practice. Occurring risks create a barrier for patients and doctors [1]. By means of the research questions the aim of this work was to find out what kind of risks a discharge letter has and what kind of measures lead to reduction or avoidance of those risks.

Material and methods

The research question was answered with a considerably literature research. A systematic search in the various databases such as PubMed, Springer Link, Google Scholar, Cochrane library and Medline revealed few recent results, especially with regard to studies published between 2005 and 2017. Studies used were mainly from Austria, Germany, Switzerland, Denmark and Norway.

Results

Risks like delayed sending of the discharge letter to doctors for further treatments, incomprehensible information in the discharge letter and unknowable information in the content of discharge letters were mentioned most commonly. A minimization of these risks is possible with the application of according measures. Examples of measures are the implementation of computer-based solutions, application of checklists and regularly advanced training.

Conclusions

Concerning this topic there is a need for further research in Austria as the current state of research is rarely available. To improve the quality of discharge letters audits and the provision of structured feedback from experienced physicians is necessary. A discharge plan detailing the physician's recommendations for the care of the patient after discharge should be prepared. Among other things, it is important to observe the regulations for discharge letters. In addition, a detailed medicinal product plan should be incorporated in the discharge letter. There must be a precise description of the dose of the medicinal product. In addition, especially in the medical education of students awareness of the importance of the issues connected with the discharge letter is missing [1].

\section{Competing interests}

The authors have no competing interests. There was no funding.

\section{References}

Bohnenkamp, B. (2016). Der Arztbrief - viel mehr als nur lästige Pflicht. Deutsches Ärzteblatt, 113(47), Zugriff am 02.11.2017 unter https:// www.aerzteblatt.de/archiv/184006/Arbeitsorganisation-Der-Arztbrief-vielmehr-als-nur-laestige-Pflicht

A8

Decision support systems in the field of pain management Marina Schreder ${ }^{1}$, Bernd Pichler ${ }^{2}$, Gerald Sendlhofer ${ }^{2,3}$

1 Department of Applied Computer Sciences, eHealth, University of Applied Sciences, Graz, Austria; ${ }^{2}$ Department for Quality and Risk Management, University Hospital Graz, Graz, Austria; ${ }^{3}$ Research Unit for Safety in Health, Division of Plastic, Aesthetic and Reconstructive

Surgery, Department of Surgery, Medical University of Graz, Graz, Austria Correspondence: Marina Schreder (marina.schreder@edu.fh-joanneum.at) Safety in Health 2018, 4(Suppl 1):A8

Key words: decision support, pain, pain management, mock up Background

Pain management is a complex and critical issue for the relief of acute pain. The occurrence of pain accounts for a lot of financial resources in annual medical expenditures, loss of quality of life and decreased worker productivity contribute to indirect costs. The introduction of pain management decision support systems is considered a potential solution for addressing the complex problems encountered in pain management. Such a system has the potential to support physicians in making their choice when it comes to the prescription of medical products. The aim of the project was to identify the possibilities and the feasibility for the implementation of such a decision support system in the field of pain management at the University Hospital of Graz. Moreover, mock ups particularly with regard to the design and structure of a decision support system will be developed.

\section{Material and methods}

Members of the operative nursing staff and physicians of three selected wards were interviewed. Each of the professional group was asked to illustrate the present workflow when it comes to pain management. Parameters like the starting point of the process, relevant documents, scales and schemes they used, the way of 
documentation of values and especially their basis of decision making were assessed.

Results

A nominal-actual comparison regarding the present pain management process was conducted in order to identify the process. The used schemes for medication prescription at the department for internal medicine, in dependence to the assessed pain-value were analyzed. The schemes were suitable and the physicians take these schemes as a kind of support, but they also highlighted that for a final decision a lot of other parameters have to be considered. The next steps are to identify and design mock ups for such a clinical decision support system.

\section{Conclusions}

Although the schemes they used were suitable for the patients at the internal medicine, a vast amount of other parameters have to be considered before the physician prescribes any drug. This could be one difficulty when it comes to the design and implementation of a decision support system.

\section{A9}

Growing together with safety - Kepler University Hospital

Nina Füreder (Nina.Fuereder@kepleruniklinikum.at)

Executive Department for Quality Management, Kepler University

Hospital, Linz, Upper Austria, Austria

Safety in Health 2018, 4(Suppl 1):A9

Key words: patient safety, attitudes, leadership, patient safety culture Background

The fourfold aim of this study was to (1) evaluate and measure attitudes concerning patient safety considering the merger of three different hospitals into one (2) investigate and compare differences in attitude based on location, profession and leadership (3) build further awareness of values, attitudes, competences and patterns behaviour to influence the workforce usefully (4) deduce necessary actions to improve the patient safety culture in the whole organisation.

\section{Materials and methods}

A quantitative survey was conducted using a standardised questionnaire. We selected a prevalent tool to assess the patient safety culture: The Hospital Survey on Patient Safety Culture Questionnaire (HSOPSC) [1] which was translated into German by ETH Zurich, Center for Organisational and Occupational Sciences (PaSKI) [2]. An adaption to local circumstances followed. In November 2017 the survey was sent online to 1191 employees randomly chosen. 174 of them answered the survey covering items like leadership, communication, teamwork, organisational learning, feedback and frequency of events reported. Data were analysed by descriptive statistics.

Results

The most positively perceived dimensions were organisational learning, $74 \%$, respectively; overall perceptions of patient safety, $74 \%$, respectively; hospital management support for patient safety, $73 \%$ The least positively perceived dimensions were hospital handoffs and transitions, $62 \%$, respectively; frequency of events reported, 62 $\%$, respectively; feedback and communication about error, $50 \%$. Overall the findings show better results concerning employees in a managing position. Furthermore we can verify more safety culture differences within profession than on location. The split of the dimension frequency of events reported into reported misses and reported near misses indicates significant fewer reported near misses. Conclusion

Considering the small response this survey assisted to identify components of culture in need of improvement, raised awareness of safety culture and evaluated effectiveness of patient safety interventions. The results demonstrate that we can make improvements in safety culture by implementing practices that support all components of an informed, safety culture. These practices must include a voluntary error reporting system that uses a standardised taxonomy to support a reporting culture. The service of a structured reporting system provides a common language that our hospital personnel can use to understand errors in the context of the interdependent structures and processes which make up their systems. Multiple strategies to communicate about and learn from errors are necessary to develop a learning culture. Prospective the organisation collects, analyses and disseminates safety-related information to act upon and learn from these data $[3,4]$.

\section{References}

1. Agency for Healthcare Research and Quality. Hospital Survey on Patient Safety Culture. [cited 2018 Jan 23]. Available from: URL: https:// www.ahrq.gov/sops/quality-patient-safety/patientsafetyculture/hospital/ index.html.

2. Center for Organisational and Occupational Sciences. Manual zum Patientensicherheitsklima-Inventar [cited 2018 Jan 23]. Available from: URL: http://docplayer.org/13752250-Manual-zum-patientensicherheitsklima-inventar-paski.html.

3. James Reason. Safety paradoxes and safety culture [cited 2018 Jan 23] Available from: URL: http://safetyhub.co.nz/wp-content/uploads/2013/09/ Safety-Paradoxes.pdf.

4. Katherine J. Jones, PT, PhD, Anne Skinner, RHIA, Liyan Xu, MS, Junfeng Sun, PhD, and Keith Mueller, PhD. The AHRQ Hospital Survey on Patient Safety Culture: A Tool to Plan and Evaluate Patient Safety Programs; 2008 [cited 2018 Jan 23]. Available from: URL: https://www.ncbi.nlm.nih.gov/ books/NBK43699/.

A10

Open disclosure after medical error: A review on economic effects Markus Holtel ${ }^{1,2}$, Stefan Pilz ${ }^{1,3}$

${ }^{1} G e s e l l s c h a f t$ für Qualitätsmanagement in der Gesundheitsversorgung (GQMG), Köln, Germany; ${ }^{2}$ Emergency Medicine, Christophorus-Kliniken Coesfeld-Dülmen-Nottuln, Nottuln, Germany; ${ }^{3}$ DR. FONTHEIM, Klinik für Psychiatrie, Psychotherapie und Psychosomatik, Liebenburg, Germany Correspondence: Markus Holtel (dr.m.holtel@online.de)

\section{Safety in Health 2018, 4(Suppl 1):A10}

Key words: Adverse events, Medical errors, Open disclosure, Patient safety

Background

In 1999, the Veteran Affairs Medical Center in Lexington/Kentucky published their 12 year-experience with systematic open disclosure after adverse events, e.g. medical errors. After losing $\$ 1.5$ million in two lawsuits, they decided to disclose them proactively. The staff notified events to the patient or his next of kin and took responsibility for the case, patients were financially compensated. In comparison with other Veteran Affairs Centers, the Lexington facility experienced no increase in total liability costs. The number of cases increased, but payments were moderate. So they considered extreme honesty the best policy.

This policy is recognized ethically laudable. However, the issue has since been discussed because of its economic effects and the fear of a maximization of malpractice claims.

Material and methods

This review examined studies, articles and official recommendations that reported the need for open disclosure, systematic disclosure strategies and their economic effects. We searched the MEDLINE, ResearchGate and Dtsch Arztebl databases. Initial search terms were "open disclosure", "adverse events" and "Offenlegung". The combined list was screened for relevant titles and abstracts and 82 titles were examined.

Results

The intriguing finding of unvaried costs induced further research. Studdert et al. made a forecast based on a survey among lawyers and risk managers in 2007. The respondents expected an increase in claims and lawsuits. Even under the assumption of $40 \%$ reduced payments, the study predicted a $72 \%$ chance that total costs would increase under routine disclosure. They saw a $34 \%$ chance of doubled costs.

This prediction did not consider the importance of trusting relationship between patient and physician. Patients tend to sue if they are suspicious about their physicians' assertions. Furthermore, a systematic disclosure gives better defence against false claims. Actually, the COPIC insurance company in Colorado reported a huge decrease in average costs in their open disclosure program. They fell from more than $\$ 78,741$ per claim to $\$ 1,820$. Overall, claims have dropped $50 \%$. 
The University of Michigan Health System compared liability claims and costs before and after implementation of a disclosure program. They found a significant decrease in claims (to 62\%) and lawsuits (to $35 \%)$. The average total liability costs dropped to $41 \%$. Since, recommendations for systematic disclosure were made e.g by the Austrian "Plattform für Patientensicherheit" and the German "Ärztekammer Nordrhein".

\section{Conclusions}

There is growing evidence, that - apart from its ethical connotations and improvement of quality - open disclosure also is an economically rational behavior.

\section{Acknowledgements}

The authors worked on this topic in the working group Kommunikation im Qualitäts- und Risikomanagement of the Gesellschaft für Qualitätsmanagement in der Gesundheitsversorgung (GQMG), Germany, which published several guidelines for communication in medical context, cf. http://www.gqmg.de/ downloads/.

\section{References}

1. Barach P, Cantor M. Adverse event disclosure: benefits and drawbacks for patients and clinicians. In: Clarke S, Oakley J, editors. Informed consent and clinician audibility. The ethics of report cards on surgeon performance. Cambridge: Cambridge University Press; 2007. p.76-91.

2. Carrillo I, Mira JJ, Guilabert M, Lorenzo S. Why an open disclosure procedure is and is not followed after an avoidable adverse event. J Patient Saf. 2017; Epub ahead of print: June 2017

3. Hingorani $M$, Wong $T$, Vafidis $G$. Attitudes after unintended injury during treatment: a survey of doctors and patients. BMJ. 1999; 318:640-641.

4. Kachalia A, Kaufman SR, Boothman R, Anderson S, Welch K, Saint S, Rogers MAM. Liability claims and costs before and after implementation of a medical error disclosure program. Ann Intern Med. 2010; 153:213-221.

5. Kraman SS, Hamm G. Risk management: Extreme honesty may be the best policy. Ann Intern Med. 1999; 131:963-967.

6. Studdert DM, Melio MM, Gawande AA, Brennan TA, Wang YC. Disclosure of medical injury to patients: an improbable risk management strategy. Health Affairs. 2007; 26:215-226.

7. Vincent C, Young M, Phillips A. Why do people sue doctors? A study of patients and relatives taking legal action. Lancet. 1994; 343:1609-1613.

\section{Competing interests}

The authors have no competing interests. There was no funding.

\section{A11}

Patients' view on the discharge process in an university hospital Alessandra Falk', Magdalena Hoffmann², Gerald Sendlhofer ${ }^{2,3}$, Gernot Brunner $^{2}$

${ }^{1}$ Institute of Nursing Science, University Hospital Graz, Graz, Austria;

${ }^{2}$ Executive Department for Quality and Risk Management, University

Hospital Graz, Graz, Austria; ${ }^{3}$ Research Unit for Safety in Health, c/o

Division of Plastic, Aesthetic and Reconstructive Surgery, Department of

Surgery, Medical University of Graz, Graz, Austria

Correspondence: Magdalena Hoffmann

(Magdalena.hoffmann@klinikum-graz.at)

Safety in Health 2018, 4(Suppl 1):A11

Key words: qualitative content analysis, patient survey, safe discharge, health literacy, focus group survey

\section{Background}

Deficient discharge of hospitalized patients can have far-reaching and serious consequences not only for the patients themselves but also for their relatives. Besides, it can also lead to financial implications for the health and social system. In a survey which was carried out by KAGes, recently dismissed patients were asked about their dismissal and various items were rated as "critical". It was the aim of this study to analyze in more detail during focus group discussions the experiences of affected patients and their relatives in order to point out possible potentials for further improvement.

Material and methods

Focus group interviews with neurological and dermatological patients $(n=21)$ were conducted using a semi-structured interview guideline. All interviews were recorded and transcribed. To analyse these data, a qualitative content analysis was conducted using the MAXQDA@ 12 encoding software.

Results

Respondents reported positive as well as negative experiences concerning their care and discharge process. Patients and their relatives experienced challenges, especially regarding information transfer. Obtained written and oral information was often poorly understood because of used medical terminology. In addition, respondents mentioned the desire of clearer and easily understandable written information, such as the doctor's discharge letter, in order to make it more "patient friendly". Another important point was often a missed dismissal interview. In addition, patients expressed positive experiences which they made during their stay and were able to express various suggestions as well as wishes.

Conclusions

The dismissal interview was a crucial theme. Respondents who said they had received such a conversation seemed much more enlightened and much happier with their stay at the university hospital. Due to difficulties of understanding, such as necessary information regarding further treatment, behaviors, or the necessary medication, patients were not dismissed fully informed which may lead to new problems, such as incorrect intake of the medication. Comprehensive and understandable information sharing lays the foundation for strengthening the health literacy of patients and restoring or improving their health. In a direct comparison, however, the respondents had more positive than negative experiences and reported positive experiences with the employees and praised them.

\section{Competing interests}

The authors have no competing interests. There was no funding.

References

none

A12

Using Manchester Triage System plus nurses' standard procedure in emergency departments may identify patients at risk earlier and reduce their risk

Norbert Bauer (norbert.bauer@lkh-hartberg.at)

Department of Internal Medicine, Hospital Hartberg, 8230 Hartberg,

Styria, Austria

Safety in Health 2018, 4(Suppl 1):A12

Key words: Manchester triage, emergency, risk, clinical management Background

Using Manchester Triage System (MTS) is standard procedure in Emergency Departments (ED) worldwide [1, 2]. Misjudgments in MTS are well known, especially in patients who are triaged in MTScategory 3 to 5 [3]. The cohort of MTS-category 3-5 accounts for about $75 \%$ of our ED patients. Patients with acute coronary syndrome (ACS) presenting with atypical clinical symptoms may have waiting periods up to 120 minutes to first medical contact (FMC). Notably, current guidelines of the European Society of Cardiology postulate an ECG within 10 minutes after FMC for patients with ACS [4]. Optimizing clinical management addressing patients at risk with atypical clinical presentation in ED deems necessary.

Methods

Recognizing that a noteworthy number of patients with cardiac diagnoses had delayed FMC applying standard MTS we adopted medical proceedings in our ED. We combined MTS with nurses' standard procedure (NSP) for all ED patients in MTS-category 3-5 including ECG recording and blood draws for predefined lab analysis immediately after triage. We denominated our proceedings MTS-2.0 (Figure 1). 
Prior to adopting MTS-2.0 and six month subsequently, we measured time intervals of all ED patients with respect to duration of treatment periods such as administration, triage, NSP or time to FMC (Figure 2). It was our intention to demonstrate, weather an ECG recording as soon as possible could identify patients at risk earlier than routine MTS.

Results

Screening 198 patients before and 250 after initiating MTS-2.0 time periods from administration to FMC declined from 66 minutes on average to 15 minutes in

MTS-category-1-patients, 24 minutes in MTS-category-2-patients and 60 minutes in MTS-category-3/5-patients respectively (Figure 2; $\mathrm{T} 1 \rightarrow \mathrm{T5}$ ). Waiting period to FMC was casual before MTS-2.0 and did not correlate with the patients' risk (T2-T5). Using MTS-2.0 all ED patients received ECG recording within 25 minutes after administration $(\mathrm{T} 1 \rightarrow \mathrm{T} 4)$. Patients in MTS-category 3-5 with suspicious ECG were immediately assigned to MTS-category 1 (Figure 2, red arrow).

A case series of several patients in MTS category-4 showed that severe diagnosis was identified up to 74 minutes earlier with MTS-2.0 than with routine MTS $(\mathrm{T} 4 \rightarrow \mathrm{T} 5)$. These diagnoses include ACS, acute myocarditis and ventricular tachycardia.

\section{Conclusions}

MTS-2.0 ascertains ECG for all patients in our ED within at least 25 minutes after administration. Early ECG is crucial to identify patients at risk with atypical clinical presentation who might receive delayed medical treatment when standard MTS is applied. MTS-2.0 may increase the quality of patients' medical care in ED.

\section{References}

1. Mackway-Jones K, Marsden J, Windle J (Hrsg). Ersteinschätzung in der Notaufnahme; Das Manchester Triage System; Deutsch von Jörg Kery und Heinzpeter Moecke. Bern, Verlag Hans Huber; 2011

2. Bauer N. Kann eine zentrale Notaufnahme in allen Krankenhäusern der steiermärkischen Krankenanstaltengesellschaft, unabhängig von der Bettenzahl, medizinisch und ökonomisch sinnvoll etabliert werden? Masterthesis im Rahmen des ULG MBA in Health Care and Hospital Management der Medizinischen Universität Graz. Graz, 2015

3. Waydhas C. Grenzen und Herausforderungen der Triage in der Notfallund Rettungsmedizin. Berlin: Springer; 2017 Nov; 20(7):574-577

4. 2017 ESC Guidelines for the management of acute myocardial infarction in patients presenting with ST-segment elevation. European Heart Journal: 2018: 39:119-177

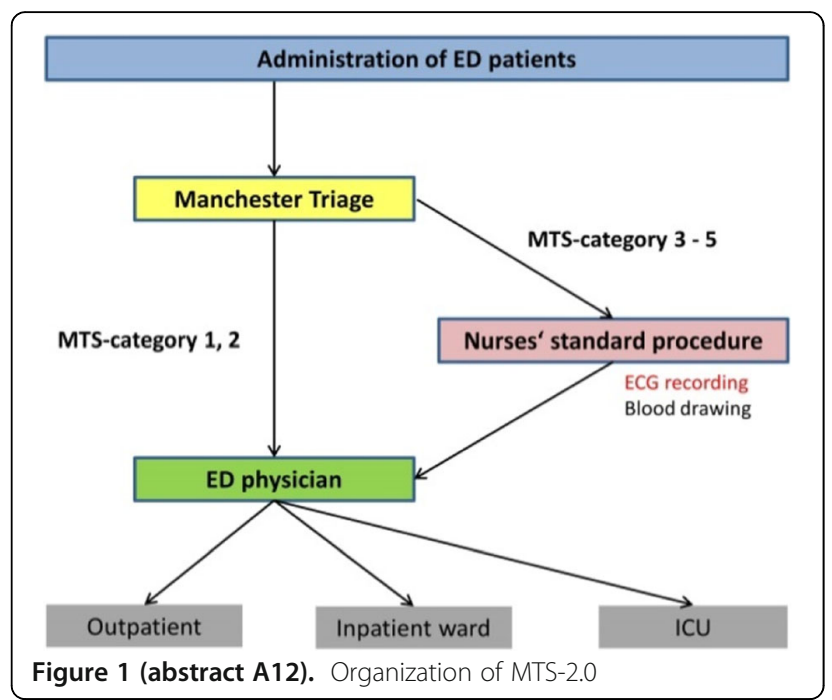

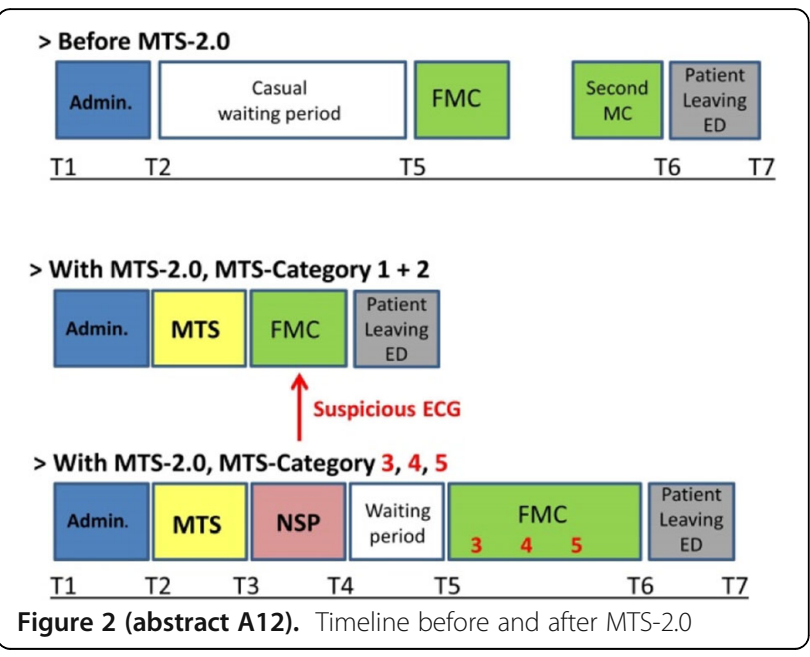

A13

Influence of standards on the results after liver transplantation Daniela Kniepeiss, Peter Schemmer

Division of Transplantation, Transplant Center Graz, Department of Surgery, Medical University of Graz, Graz, Austria

Correspondence: Peter Schemmer (peter.schemmer@medunigraz.at) Safety in Health 2018, 4(Suppl 1):A13

Key words: quality management, standards Background

Standards are internationally accepted Quality Control-Mechanisms and are used to optimize how resources are used. The objective was to observe how the introduction of perioperative standards would influence both the quality of the results and the usage of resources.

Material and methods

The checklist for patient examinations prior to liver transplantation was established and optimized. In addition interdisciplinary indication conferences, perioperative patient developments, therapy standards, 24/7 pathological - call service, utilization of innovative OP- techniques as well as mortality- and morbidity-conferences were applied. The influence of these measures on the quality of the results and the resource usage has been first documented and then evaluated and compared with publications.

Results

The quality of the results showed a clear improvement to a level that is equivalent to a high-volume transplant center. There was a positive influence on patients time spent both in the intensive care unit and hospital overall as well as morbidity and mortality.

Conclusions

A continuous and well-structured revision of medical content of transplant programs will result in improved patient care.

\section{Competing interests}

The authors have no competing interests. There was no funding.

\section{A14}

Can fall and pressure ulcer guidelines improve nursing care in hospitals? A cross-sectional study

Doris Eglseer, Manuela Hödl, Christa Lohrmann

Institute of Nursing Science, University Hospital Graz, Graz, Austria

Correspondence: Doris Eglseer (doris.eglseer@medunigraz.at)

Safety in Health 2018, 4(Suppl 1):A14 
Key words: fall, pressure ulcer, guideline, nursing interventions Background

Falls and pressure ulcers (PU) are nursing care problems that can negatively affect patient safety in hospitals. Evidence-based guidelines with regard to this topics aim at preventing falls and the development of PU during the hospital stay. The aim of this study was to investigate the association between the presence of a guideline with regard to falls and PUs and the nursing interventions conducted in patients admitted to these hospitals.

Materials and methods

The International Prevalence Measurement of Care Problems (LPZ) is an annually conducted cross-sectional multi-center study. To answer the research question, 33 Austrian hospitals and 2824 patients that participated in the measurement on Nov. $14^{\text {th }} 2017$ were included in the analysis. Hospital nurse directors were asked if there is a guideline available in the respective hospitals for falls and PUs. Nursing interventions were collected in every patient that was admitted at the wards of the participating hospitals at the day of data collection and agreed to participate. Data were collected by asking the patients as well as using patient records. Results

In $78.8 \%$ and $97 \%$ of the participating hospitals there was a guideline for the prevention and treatment of PUs and fall prevention respectively available. In hospitals with a PU guideline, nurses conducted significantly more interventions to prevent the development of PUs, e.g. they used more often reactive support surfaces, seating support surfaces, moisturizer to protect the skin and mobilized patients more often. In general, the results showed that patients in hospitals with a guideline for preventing falls received more nursing interventions than patients in hospitals without a guideline. These patients in hospitals with a guideline for preventing falls significantly received more therapeutic exercises; nurses evaluated more often the current medication and eyesight/vision and conducted more often safety adaptations of the environment.

\section{Conclusions}

The data of the current study shows that the presence of guidelines significantly increases the number of nursing interventions to prevent PUs and falls in Austrian hospitals, which might also contribute to increased patient safety in these hospitals.

\section{A15}

Designing Transitions: Optimization options for the transfer of patients between nursing homes and acute-care hospitals Brigitta Schatz ${ }^{1}$, Herbert Rettl ${ }^{2}$, Eva Maria Jabinger

${ }^{1}$ fhg - Zentrum für Gesundheitsberufe Tirol GmbH, University of Applied Sciences Tyrol, Innsbruck, 6020, Austria; ${ }^{2}$ Landeskrankenhaus Hall in Tirol, Tirol Kliniken GmbH, Hall/Tyrol, Innsbruck, 6060, Austria

Correspondence: Brigitta Schatz (schatz.brigitta@gmail.com)

Safety in Health 2018, 4(Suppl 1):A15

Key words: Optimization methods, transfer management, acute care hospital, long-term care facility

Background

The strict separation between the inpatient and the outpatient sector in the Austrian health care system causes interfaces that can lead to a lack of information and other problems (Greiling, Dudek). Due to the demographic and social-political development, the interface between long-term care facilities and acute-care hospitals is particularly important (Milisen, Arends). The transition of patients from one institution to another must be improved to increase their safety and satisfaction. Methods

The aim of this thesis is to show which methods are suitable for the optimization of the transition of patients between long-term care facilities and acute-care hospitals. For this purpose, a structured literature search in online databases was carried out first to identify possible optimization methods. In order to evaluate these methods from the angle of practice, interviews were conducted with nurses. The interviews were based on a partially structured interview guideline. The basis for its preparation was the theoretical discussion in the first part of the research. The evaluation of the data collected in the course of the interview was based on the summarizing content analysis according to Mayring.
Results

Six possible optimization methods were identified from ten studies, which corresponded to the inclusion criteria of the research (see Table 1). In the course of the work, these methods will be used as possible optimization methods the transfer of patients between nursing homes and acute-care hospitals.

Three nurses from nursing homes and three from acute-care hospitals were asked to assess the corresponding methods from a practical point of view. Checklist received the highest approval in the overall assessment of the methods. Cooperation and networking was evaluated in a similar way. The result of the evaluation according to the overall impression is supported by the number of mentioned advantages and disadvantages to each optimization methods. In addition to the theoretical examination of the topic, a case study was carried out in the Landeskrankenhaus Hall in Tirol. It describes the implementation of these two of the identified methods in more detail.

\section{Conclusion}

This study is meant to provide an overview of possible methods to improve the transition of patients between long-term care facilities and acute-care hospitals. The selection of the appropriate method must be made according to the given framework conditions. Based on the results of the study, Checklist as well as Cooperation and Networking can be highly recommended.

\section{References}

1. Greiling, Michael; Dudek, Matthias (2009): Schnittstellenmanagement in der Integrierten Versorgung. Eine Analyse der Informations- und Dokumentationsabläufe. 1. Aufl. s.l.: Kohlhammer Verlag. Online verfügbar unter https://www.kohlhammer.de/wms/instances/KOB/appDE/Krankenhaus/ Krankenhausmanagement/Schnittstellenmanagement-in-der-IntegriertenVersorgung-978-3-17-019152-5/.

2. Milisen, Koen; Arends, Helmut (Hg.) (2004): Die Pflege alter Menschen in speziellen Lebenssituationen. Modern, wissenschaftlich, praktisch. Berlin: Springer.

Table 1 (abstract A15) . Optimization methods with number of studies identified (source: from own results)

\begin{tabular}{ll}
\hline Optimization methods & Number of studies \\
\hline IT solution & 3 \\
Checklist & 2 \\
Cooperation and Networking & 2 \\
Standardized-transfer-reports & 1 \\
Training of employees & 1 \\
Process-optimization & 1 \\
\hline
\end{tabular}

\section{A16}

Optimization of a Clinical Process "MR Safety"

Silvia Franzelin, Christian Kremser

University of Applied Sciences, Master degree programme of Quality and Process Management in the Health Care Sector 2017, Innsbruck, Austria

Correspondence: Silvia Franzelin (Silvia.franzelin@tirol-kliniken.at) Safety in Health 2018, 4(Suppl 1):A16

Key words: MR imaging, implants, safety, standards, prozess analysis Background

Magnetic Resonance Tomography (MRT) is a complex operation involving interpersonal processes. During an MRI examination, patients are exposed to a strong static magnetic field, fast changing magnetic field gradients and strong high frequency fields, which can impose danger to patients with certain implants. It was the purpose of this work to analyze and model the current state of the MR safety process at our hospital regarding implants and to derive the potential for optimization by comparing with an MR safety process in a hospital of about the same size. 


\section{Material and Methods}

After literature research and expert interviews, the MR safety process was modelled applying the Business Process Model and Notation (BPMN) developed by the BOC group and a risk estimation regarding the MR safety for patients with implants was performed. Subsequently, using a questionnaire covering 20 implant classes, data regarding MR safety of implants were collected over a period of two weeks. The results were the analyzed by descriptive statistics using SPSS and key parameters for the MR safety process were identified. Where possible the results were compared to a similar study for a comparable hospital. Results

A total of 674 questionnaires were evaluated. In 235 cases (34.9\%), patients had implants, however, in only two cases $(0.3 \%)$ the MR examination could not be performed. In the majority of cases, obtaining information regarding the MR compatibility of an implant took less than $3 \mathrm{~min}$ and in very seldom cases (4 cases in two weeks) up to $45 \mathrm{~min}$. Long evaluation times typically resulted for new or rare implants. The direct comparison to a similar hospital regarding MR safety procedures essentially revealed differences in the management of appointment requests and appointment booking. In case of appointment handling by administrative staff, which does not check the implant status, implants typically have to be evaluated at the time of patient arrival, whereas in case of appointment handling by MR specialized technicians implants can already be evaluated at an earlier stage.

Conclusions

Potential for improvements regarding MR safety processes could be identified for the process of appointment handling. Further, permanently raising the awareness among referring physicians regarding MR safety of implants and continuous interdisciplinary trainings can be identified as important measures to improve MR safety. Description and presentation of processes makes interdisciplinary cooperation more transparent and could lead to an increase of efficiency regarding MR safety.

\section{A17}

The result matters - Quality measurements by means of quality registries

Martina Lerchner, Matthias Gruber, Reinhard Kern

Austrian Public Health Institute (Gesundheit Österreich GmbH), Federal

Institute for Quality in the Health Care System (Bundesinstitut für

Qualität im Gesundheitswesen), Vienna, Austria

Correspondence: Martina Lerchner (martina.lerchner@goeg.at)

Safety in Health 2018, 4(Suppl 1):A17

Key words: Quality measurements; quality registries; nationwide database; hospital quality improvement; patient safety BACKGROUND

The Austrian Public Health Institute (GÖG) develops and manages currently 7 quality outcome registries. Quality outcome registries aim to provide a comparative description of care received and of treatment results. They are also a useful tool to identify potential areas for future activities related to quality improvement and patient safety. The maintenance of the registries is required by law [1-3].

\section{MATERIALS AND METHODS}

Data are collected either via a web-application or decentrally in the hospitals (periodical transmission). Data of almost all registries undergo routine plausibility checks, comparing them with DRG (Diagnosis-related groups) -based hospital data and mortality data provided by Statistic Austria. Descriptive- and benchmark analyses are carried out regularly by the Austrian Public Health Institute. Figure 1 shows the process of quality outcome measurements. Table 1 gives an overview about all 7 quality outcome registries. RESULTS

All participating hospitals compare their own results to the national average and to other hospitals on a pseudonymised basis. As an outcome of this process hospitals define their own measures to improve quality of treatment and care (e. g. comprehensive training concerning surgical techniques, optimization of structures in hospitals). A positive aspect concerning patient safety is, that better quality leads e. g. to fewer complications and lower mortality rates. Figure 2 shows exemplary a positive evaluation result: the percentage of Lysis rate concerning selected patients increased over the past years. Results are not open to the public, but shared only with participating hospitals and clients (e. g. Ministry of health).

\section{CONCLUSIONS}

Registries can serve as a comprehensive and high quality nationwide database, describing the treatment and care of patients in hospitals. With a standardised quality improvement process and a high participation rate, quality outcome registries contribute significantly to nation-wide quality assurance of medical therapy and especially to the improvement of patient safety.

\section{REFERENCES}

1. Bundes- Zielsteuerungsvertrag https://www.bmgf.gv.at/cms/home/ attachments/2/8/6/CH1443/CMS1501063225561/ zielsteuerungsvertrag_auf_bundesebene_2017-2021.pdf

2. Bundesgesetz über die Gesundheit Österreich GmbH (GÖGG) https:// www.ris.bka.gv.at/GeltendeFassung.wxe?Abfrage $=B$ undesnormen\&Gesetzesnummer $=20004884$

3. Bundesgesetz betreffend Medizinprodukte (Medizinproduktegesetz MPG) https://www.ris.bka.gv.at/GeltendeFassung.wxe?Abfrage=Bun desnormen\&Gesetzesnummer $=10011003$

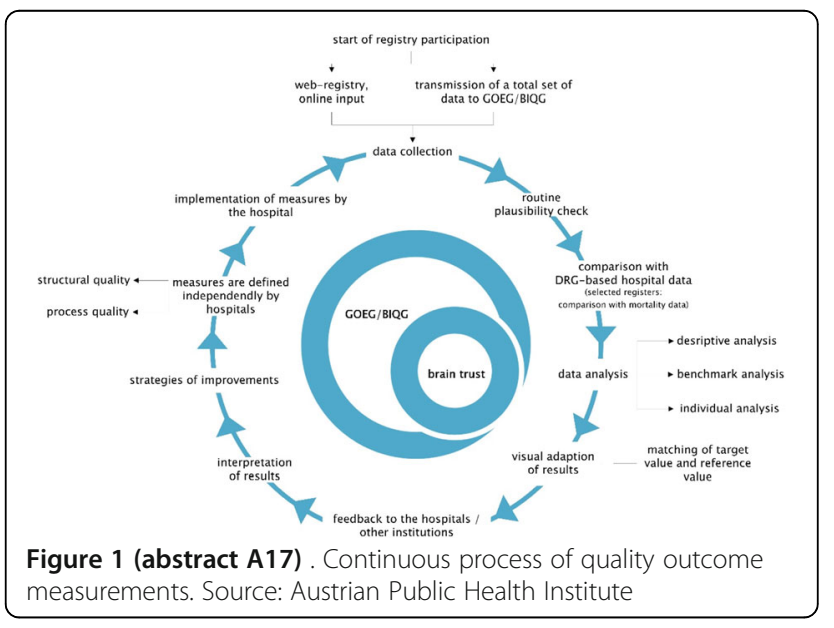

Table 1 (Abstract A17) . Quality outcome registries currently maintained by the Austrian Public Health Institute

\begin{tabular}{|c|c|c|}
\hline Registries & Coverage & $\begin{array}{l}\text { Selected Indicators } \\
\text { (examples) }\end{array}$ \\
\hline Stroke Unit Registry & 35 of 37 Austrian Stroke Units & $\begin{array}{l}\cdot \text { - NIH Stroke Scale } \\
\text { - time passed between } \\
\text { event \& hospitalization }\end{array}$ \\
\hline $\begin{array}{l}\text { Quality measurement of } \\
\text { hip \& knee endoprosthesis }\end{array}$ & all Austrian acute care hospitals & $\begin{array}{l}\text { - revision count } \\
\text { - complications }\end{array}$ \\
\hline $\begin{array}{l}\text { Adult cardiac surgery } \\
\text { Registry }\end{array}$ & 9 of 9 Austrian cardiac surgeries & $\begin{array}{l}\text { - standardized mortality } \\
\text { rate } \\
\text { - postoperative } \\
\text { complications }\end{array}$ \\
\hline $\begin{array}{l}\text { Register for pacemakers, } \\
\text { ICD and loop recorders }\end{array}$ & $\begin{array}{l}\text { mandatory data transfer } \\
\text { according to the Medical } \\
\text { Devices Act }\end{array}$ & $\begin{array}{l}\text { - reasons for } \\
\text { explantation } \\
\text { - complications }\end{array}$ \\
\hline $\begin{array}{l}\text { Neonatology Outcome - } \\
\text { Registry }\end{array}$ & $\begin{array}{l}\text { currently } 22 \text { acute care hospitals } \\
\text { and nearly } 5.400 \text { data sets }\end{array}$ & $\begin{array}{l}\text { - Cerebral palsy per } \\
\text { birth- weight group } \\
\text { - mortality }\end{array}$ \\
\hline $\begin{array}{l}\text { Registry for radical } \\
\text { cystectomies }\end{array}$ & $\begin{array}{l}\text { shut down; } 24 \text { acute care } \\
\text { hospitals and nearly } 600 \text { data } \\
\text { sets }\end{array}$ & $\begin{array}{l}\text { - All radical } \\
\text { cystectomies; } \\
\text { - re-hospitalisation }\end{array}$ \\
\hline $\begin{array}{l}\text { Quality assurance in } \\
\text { vascular surgery }\end{array}$ & currently developed & $\begin{array}{l}\text { - complications } \\
\text { - mortality }\end{array}$ \\
\hline
\end{tabular}

Source: Austrian Public Health Institute 


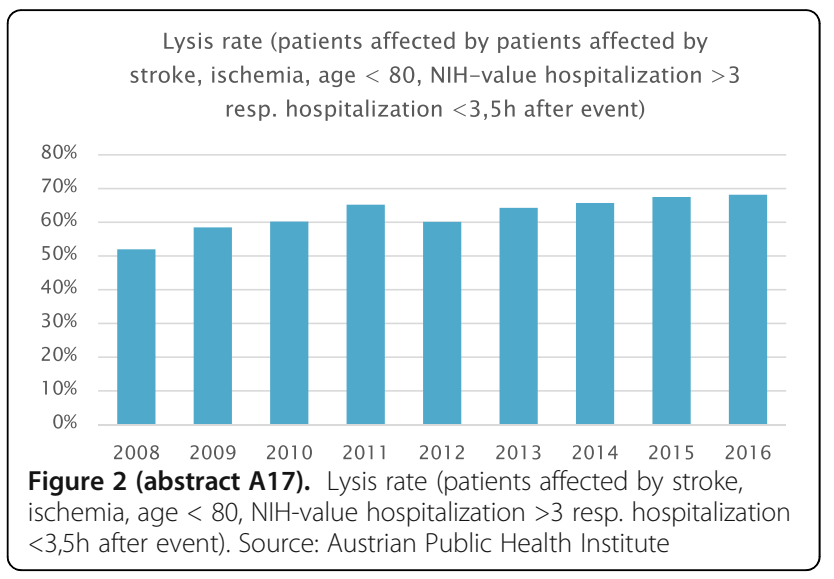

A18

Information Platform on Medication Safety within the Austrian Health Portal

Heidi Stürzlinger, Isabella Röhrling, Sabine Vogler, Eva Pilz, Alexander Eisenmann

Austrian Public Health Institute (Gesundheit Österreich GmbH), Vienna, Austria

Correspondence: Heidi Stürzlinger (heidi.stuerzlinger@goeg.at)

Safety in Health 2018, 4(Suppl 1):A18

Key words: Medication safety, prescription, drug, drug interaction, dosage

BACKGROUND

Doctors are confronted with a great deal of information and requirements when prescribing drugs. Drug interactions or the choice of the right dosage pose a major challenge, especially for older people or children; guidelines and information on this often are not "ready at hand". The aim of this project, commissioned by the Austrian Ministry of Health and conducted by Gesundheit Österreich $\mathrm{GmbH}$, is to provide doctors with fast access to practical tools and important evidence for safe and efficient prescription of medicines via an Internet platform and to support them in their individual training. An important sub-target is the compact bundling of information without duplicating existing offers.

Material and Methods

The project team searches for information resources and tools for the safe and rational use of medicines - in general as well as on key topics. First priority is given to material related to Austria. Further material, preferably in German or English language, may be included if relevant. After screening, selection, evaluation and preparation of the information resources or tools, relevant medical and pharmaceutical experts from various areas of the health care system discuss the contents, structure and presentation on the website in workshops. The platform is publicly accessible (Informationsplattform Arzneimittelsicherheit [https://www.gesundheit.gv.at/gesund heitssystem/professional/arzneimittelsicherheit/inhalt]), embedded in the Austrian Health Portal [https://www.gesundheit.gv.at/] and bound to its quality criteria.

RESULTS

The platform was launched in February 2017, with the first key topic "polypharmacy \& elderly". The second key topic "medicines for paediatric use" will be launched in February 2018. Figure 1 shows the landing page. The platform is available in German only. The general section gives relevant information on the pharmaceutical system in Austria, including laws and regulations, responsible institutions and relevant tools. The key topics include, for example, a clearly structured selection of tools and lists for drug evaluation in polypharmacy or databases for pediatric dosages, relevant guidelines and links to thematic literature.

\section{CONCLUSIONS/OUTLOOK}

The aim of a target-group-oriented and user-friendly preparation of key tools and information resources has successfully been implemented and received positive feedback from all parties involved. The continuous updating of the contents is seen as an important success factor. An integration into continuing medical education of doctors is planned.

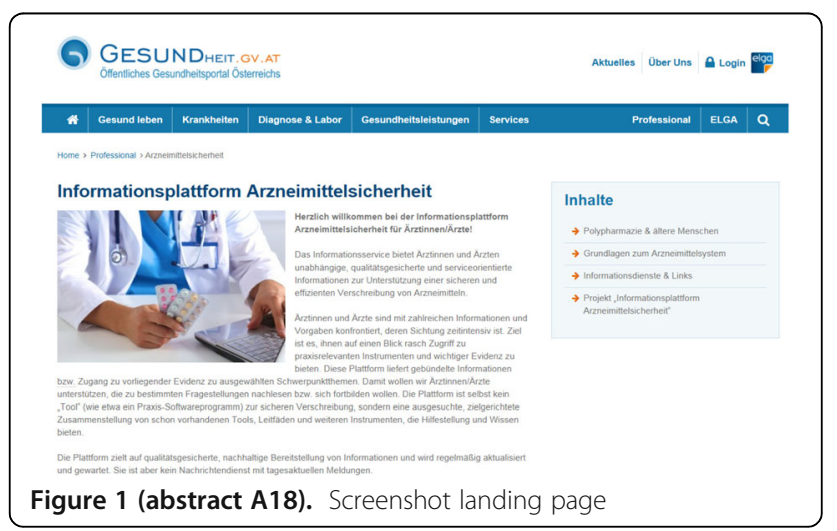

Publisher's Note

Springer Nature remains neutral with regard to jurisdictional claims in published maps and institutional affiliations. 